Original research article

Section: Food Quality and Functionality

\title{
Sprouted and Non-Sprouted Chickpea Flours: Effects on Sensory Traits in Pasta and Antioxidant Capacity
}

\author{
Joseph A. Bruno ${ }^{1}$, David W. Konas ${ }^{2}$, Evan L. Matthews ${ }^{3}$, Charles \\ H. Feldman ${ }^{1}$, Kate M. Pinsley ${ }^{2}$, Adrian L. Kerrihard ${ }^{*}$. \\ ${ }^{1}$ Department of Nutrition and Food Studies, College of Education and Human Services, \\ Montclair State University, 1 Normal Ave, Montclair, NJ 07043, USA \\ ${ }^{2}$ Department of Chemistry and Biochemistry, College of Science and Mathematics, \\ Montclair State University, 1 Normal Ave, Montclair, NJ 07043, USA \\ ${ }^{3}$ Department of Exercise Science and Physical Education, College of Education and Human Services, \\ Montclair State University, 1 Normal Ave, Montclair, NJ 07043, USA
}

Key words: chickpea, sensory traits, germination, antioxidants, phenolics

Chickpea flour, mainly from non-sprouted chickpeas, serves as an alternative to wheat flours. Sprouting legumes may improve antioxidant potential, but sensory effects of sprouting chickpeas for flour are largely unknown. This study evaluated sensory effects of up to $40 \%$ substitution of Sprouted Chickpea Flour (SCF) and Non-Sprouted Chickpea Flour (NSCF) in pasta. Total phenolics and antioxidant potential (as Trolox equivalency) of the flours were also assessed. Results showed phenolic contents and antioxidant potential were significantly higher in SCF than NSCF. By descriptive analysis, chickpea flour levels corresponded with decreases in chewiness and pasta flavor, and increases in mushiness, grittiness, bitterness, and earthiness. Effects on bitterness, earthiness, and pasta flavor were greater with SCF than NSCF. By consumer assessment, $20 \%$ SCF did not exhibit significantly lower overall hedonic measures than the other samples. With attention given to possible organoleptic challenges, SCF may warrant consideration as a more antioxidant-rich alternative to NSCF.

\section{INTRODUCTION}

Chickpea flour has been recently increasing in prominence as an alternative to wheat flour, with Future Market Insights [FMI] forecasting the global chickpea flour market to likely surpass a valuation of five billion USD by the end of 2026 [FMI, 2018]. The market for chickpea flour includes direct sales to consumers (e.g. as bags of flour for home use) and for use in product manufacturing (e.g. commercial breads and pastas made with chickpea flour). At present, non-sprouted chickpea flour (NSCF) is much more common than sprouted chickpea flour (SCF) in commercial production, but there is evidence that sprouting legumes such as chickpeas prior to flour production may improve antioxidant potential and other nutritional attributes [Devi et al., 2015; Gunashree et al., 2014]. As with other gluten-free flours, the direct substitution of chickpea flours for wheat flour can present sensory challenges, and the differences between SCF and NSCF in sensory properties are not well understood [Melini et al., 2017; Zafar et al., 2015]. Therefore, the nutritional and sensory properties of SCF, specifically

\footnotetext{
* Corresponding Author: Tel.: (707) 495-2103;

E-mail: kerriharda@montclair.edu (A. Kerrihard)
}

regarding comparison with NSCF, is a matter in need of further investigation.

Chickpea flours may appeal to consumers for their perceived nutritional benefits and lack of gluten. Chickpeas are pulse legumes, which have received substantial attention for their apparent healthfulness [Świeca et al., 2013]. Currently, many nutritionists and dietitians are recommending increased consumption of pulse legumes [Venn et al., 2010]. Furthermore, the American Diabetes Association [Polak et al., 2015] and the American Heart Association [Stone et al., 2014] recommend pulses for better cardiovascular health and blood glucose control, as well as for a healthy source of protein and starch [Świeca et al., 2013]. Chickpeas, specifically, are calculated to have $25.3-28.9 \mathrm{~g} 100 \mathrm{~g}$ protein content [Khattak et al., 2007] and have been noted for their historic role in the Mediterranean diet [Gupta et al., 2017]. Chickpeas are also known to be good sources of a large variety of vitamins, minerals, and polyphenolics [Bouchenak \& Lamri-Senhadji, 2013; Segev et al., 2011; Khattak et al., 2007].

Multiple studies have shown improvements in the nutritional properties of legumes as an effect of sprouting [Ramesh \& Swami, 2016; Devi et al., 2015; Nakitto et al., 2015; Masood et al., 2014]. Noted effects have included increases in minerals, aspartic acid, folic acid, and vitamins, with decreases 
in crude fat, crude carbohydrate, and antinutrients such as trypsin inhibitors and $\alpha$-amylase inhibitors [Devi et al., 2015; Gao et al., 2015; Gunashree et al., 2014; Świeca et al., 2013]. A recent study concluded that sprouting chickpeas increased the content of protein, minerals, and fiber, while decreasing fat content [Masood et al., 2014].

It has been shown that sprouting can increase the total antioxidant activity or phenolic contents of legumes [Ramesh \& Swami, 2016; Gharachorloo et al., 2013; Świeca et al., 2013]. A recent study focusing on chickpea flour showed SCF to positively influence brachial artery flow mediate dilation in vivo [Enrique et al., 2018].

Wood [2009] studied consumer acceptability of spaghetti fortified with NSCF at levels up to $30 \%$. Although pasta firmness decreased with increases in chickpea fortification, the study concluded that non-sprouted chickpea-fortified spaghetti was acceptable to consumers [Wood, 2009]. Another study found that adding small amounts of NSCF to wheat flour created a dough with higher strength and added elasticity [Sabanis et al., 2006]. This improvement was noted at substitution levels ranging from $5-20 \%$, but there was noted quality deterioration when more than $30 \%$ was added to the flour. An investigation of substituting chickpea flour in cracker production found that higher substitution levels were associated with increased leguminous odor and bitter taste [Kohajdová et al., 2011]. Despite the sensory challenges of chickpea flour substitution, the practice has proved viable, as evidenced by the growing market for chickpea flour and chickpea-flour rich products [FMI, 2018]. Efforts to improve the sensory quality of chickpea-flour products has included optimization of substitution levels, and addition of hydrocolloids such as pectin and gums, to name a few [Padalino et al., 2015]. How these sensory challenges would be different when using SCF rather than NSCF is currently unknown.

With the substantial rise in chickpea flour use, there has been an increasing need to further understand the differences between SCF and NSCF in regard to nutritional and sensory properties. The objectives of this study were to compare the total phenolics contents and antioxidant capacity between these two flours, and to determine the effects of both flour types on sensory properties and consumer acceptability when used in pasta production.

\section{MATERIALS AND METHODS}

\section{Chickpea sprouting}

Dry Goya chickpeas were sprouted in the Food Science Laboratory at Montclair State University (Montclair, NJ, USA) using a protocol described in a previous study [Khattak et al., 2007]. In brief, the chickpeas were submerged in deionized water for $18 \mathrm{~h}$, and then placed in a porous colander and rinsed with deionized water three times per day for six days. During this period, the chickpeas were placed in a $0.56^{\circ} \mathrm{C}$ refrigerator overnight to prevent bacterial growth [Kumar et al., 2006]. At the conclusion of the incubation, chickpeas that had not visibly sprouted were discarded and the sprouted chickpeas were placed into an Excalibur Food Dehydrator (Excalibur, Sacramento, CA, USA) at $49^{\circ} \mathrm{C}$ for $15 \mathrm{~h}$.

\section{Sprouted and non-sprouted chickpea flour production}

For both flour types, Goya chickpeas were ground into flour in a Vitamix Blender (Vitamix, Cleveland, Ohio, USA). SCF utilized chickpeas that had undergone the sprouting procedure described above, and NSCF utilized untreated dry chickpeas.

\section{Total phenolics content}

Total phenolics content (TPC) was evaluated in triplicate for each chickpea flour type in accordance with the methodology described by Singleton et al. [1999] with minor modifications. For each assessment, $5 \mathrm{~g}$ of dry flour sample was extracted twice at room temperature for $15 \mathrm{~min}$ using gentle shaking and sonication in $40 \mathrm{~mL}$ of $4: 1(\mathrm{v} / \mathrm{v})$ acetone/water. The extracts were combined and the solvents were removed under reduced pressure by rotary evaporation at $40^{\circ} \mathrm{C}$ followed by high vacuum at room temperature. The resulting material was dissolved in methanol. Assessments were performed in cuvettes containing water, methanolic sample, commercial Folin-Ciocalteu reagent, and saturated $\mathrm{Na}_{2} \mathrm{CO}_{3}$, with a final volume of $1.0 \mathrm{~mL}$ (using solution without sample extract as a blank). Each cuvette was incubated at room temperature for $1 \mathrm{~h}$ before recording the absorbance at $750 \mathrm{~nm}$ vs. a blank containing no sample. The instrument used was a Cary 300 Bio UV/Visible Spectrophotometer (Agilent Technologies, Santa Clara, California, USA). Absorbance values were correlated to the best fit line of a standard curve constructed using $0.85-8.50 \mu \mathrm{g} / \mathrm{mL}$ gallic acid and reported as $\mathrm{mg}$ gallic acid equivalents (GAE)/g flour.

\section{Trolox antioxidant activity}

DPPH radical scavenging activity was determined in quadruplicate for NSCF and SCF by measurement of Trolox Antioxidant Activity (TAA). The protocol was as described in Brand-Williams et al. [1995] with minor modifications. Hydrophilic fractions of $1.25 \mathrm{~g}$ of flour were extracted with $4: 1$ (v/v) acetone/water solvents, with the solvents subsequently removed by evaporation under reduced pressure. The fractions were dissolved in 99.7:0.3 (v/v) water/formic acid to a final volume of $7.5 \mathrm{~mL}$. $10 \mu \mathrm{L}$ of sample solution were added to $290 \mu \mathrm{L}$ of DPPH solution $(0.10 \mathrm{mM}$ prepared in $4: 1(\mathrm{v} / \mathrm{v})$ metha$\mathrm{nol} / \mathrm{H}_{2} \mathrm{O}$ ) in wells of a VersaMax ELISA Microplate Reader (VersaMax, Sunnyvale, CA USA). Absorbance was recorded at $517 \mathrm{~nm}$ following $30-\mathrm{min}$ incubation at $25^{\circ} \mathrm{C}$. The values were plotted to a standard curve constructed using solutions of $0-5 \mathrm{mM}$ Trolox in 1:1 (v/v) acetone/water. TAA values were reported as mmol Trolox equivalent/100 $\mathrm{g}$ flour.

\section{Pasta preparation}

Five different flour compositions were prepared for use in pasta sample preparation, representing incorporations of NSCF and SCF into semolina flour at a range of concentrations (100\% semolina, 20\% NSCF, 40\% NSCF, 20\% SCF, and $40 \% \mathrm{SCF}$ ). These substitution levels were chosen partially with consideration of a prior investigation by Wood [2009], but also with consideration of our own preliminary trials that determined substantial texture changes at substitution levels greater than $40 \%$. The semolina flour was "Bob's Red Mill Semolina Flour" (Bob's Red Mill, Milwaukie, OR, USA). 
The production of dough from flour was consistent for all sample types, accomplished by combining $400 \mathrm{~g}$ of the flour mixture with $118 \mathrm{~mL}$ of water. The flour mixture was mixed with water until it formed a solid dough. This dough was then kneaded and wrapped in plastic wrap and left to sit at room temperature for $10 \mathrm{~min}$. The dough was then formed into smaller balls and placed into a Kitchen Aid Gourmet Pasta Press attachment of a Kitchen Aid machine (Benton Harbor, MI, USA) and used to make fusilli pasta. The pasta was refrigerated at $0.56^{\circ} \mathrm{C}$ for $24 \mathrm{~h}$. Prior to the serving of samples, $173 \mathrm{~g}$ portions of pasta were placed in $710 \mathrm{~mL}$ of boiling water for $5 \mathrm{~min}$ and drained with a colander.

\section{Descriptive analysis}

A modification of the Spectrum ${ }^{\mathrm{TM}}$ Descriptive Analysis Method was used to determine textural attributes of pasta "chewiness", "mushiness" and "grittiness"; taste attributes of "saltiness", "sweetness", "bitterness"; and flavor attributes of "earthiness" and "pasta flavor". Panelists $(n=8)$ marked assessments on $15 \mathrm{~cm}$ lines, where the leftmost side was labeled as "not perceptible" and the rightmost side was labeled as "high intensity" [Meilgaard et al., 1999]. The scores were measured by a ruler and reported on a 0 to 1 scale of intensity.

Participants were recruited from the students and employee population of Montclair State University (Montclair, NJ, USA). The panelists received two training sessions, consisting of calibration to the intensity of listed sensory traits according to sensory standards [Meilgaard et al., 1999].

Panelists assessed each of the five pasta types in triplicate. Assessments consisted of three separate sessions, with five samples evaluated during each testing session. Samples were pre-coded with 3-digit random numbers and evaluated in a counterbalanced order. Assessments took place under white light by panelists seated in individual booths. The uncooked pasta samples were always freshly prepared the day prior to assessment and then cooked immediately prior to assessment.

\section{Consumer assessment}

Consumer assessment followed ASTM methodology with minor modifications [ASTM, 2011]. The assessment was completed by 108 untrained panelists recruited from the students and employee population of Montclair State University (Montclair, NJ, USA). Each panelist evaluated all five pasta samples. Samples were pre-coded with 3-digit random numbers and prepared and presented as described above for the descriptive analysis.

Panelists were asked to rate each sample for their hedonic assessments of appearance, texture, flavor, and overall likability. The panelists were presented a 7-point hedonic scale ranging from "dislike extremely" to "like extremely". Following self-reporting by the panelists, investigators converted panelists' responses on the lingual 7-point scale into a numeric scale ranging from zero ("dislike extremely") to six ("like extremely").

\section{Statistical analysis}

All statistical analyses were performed with IBM SPSS Statistics for Windows (Version 24.0, IBM Corp, Armonk, NY, USA). TPC and TAA values were each evaluated for
TABLE 1. Antioxidant assessments: total phenolics content and Trolox antioxidant activity of NSCF and SCF.

\begin{tabular}{l|c|c|c}
\hline Assessment & $\mathrm{NSCF}^{\mathrm{a}}$ & $\mathrm{SCF}^{\mathrm{a}}$ & $\begin{array}{c}\% \\
\text { Difference }^{\mathrm{b}}\end{array}$ \\
\hline TPC (mg GAE/g flour; $\mathrm{n}=3)$ & $7.3 \pm 0.08^{\mathrm{B}}$ & $8.4 \pm 0.22^{\mathrm{A}}$ & $+15.1 \%$ \\
$\begin{array}{l}\text { TAA (mmol Trolox } \\
\text { equivalent/100 g flour; } \mathrm{n}=4)\end{array}$ & $2.06 \pm 0.04^{\mathrm{B}}$ & $2.36 \pm 0.03^{\mathrm{A}}$ & $+14.6 \%$ \\
\hline
\end{tabular}

aMean \pm SD; NSCF $=$ Non-Sprouted Chickpea Flour; SCF $=$ Sprouted Chickpea Flour; ${ }^{b}$ Percentage difference in SCF value vs. NSCF value. Values followed by the same superscript capital letter within a row were not significantly different from one another $(\alpha=0.05)$ according to t-test via SAS software.

significant differences between samples by unpaired t-test $(\alpha=0.05)$. Significant differences between samples for descriptive analysis and consumer assessment results were determined by one-way ANOVA with Tukey's Studentized Range test $(\alpha=0.05)$.

Multivariable linear regression models were produced by modeling NSCF and SCF levels as independent variables vs. each of the assessed descriptive traits. The stepwise linear regression function in SPSS was implemented with an exclusion criteria of $\alpha=0.10$. If no variable exceeded a $p$-value of 0.10 , no model was reported for that measure.

\section{RESULTS AND DISCUSSION}

\section{Antioxidant assessments: total phenolics content and Trolox antioxidant activity}

The results of the TPC and TAA evaluations are shown in Table $1 . \mathrm{SCF}$ (8.4 mg GAE/g flour) had significantly greater TPC values than NSCF (7.3 mg GAE/g flour; $p=0.0013)$. $\mathrm{SCF}$ (2.36 mmol Trolox equivalent/100 g flour) also had significantly greater TAA values than NSCF (2.06 mmol Trolox equivalent/100 g flour; $p<0.0001)$.

Notably, the observed increases associated with sprouting were extremely similar for both TPC and TAA (15.1\% and $14.6 \%$, respectively). These observations also correspond very closely to the $13.6 \%$ increase in the total antioxidant capacity (assessed by phosphomolybdenum method) following sprouting of chickpeas observed recently by Ramesh \& Swami [2016].

A prior study has determined isoflavonoid content and diversity to increase dramatically (i.e. up to 500\%) within chickpeas during germination [Wu et al., 2012], so this may well be substantially contributing to our observed increases in phenolic contents and antioxidant activity. Although the specific in vivo effects of increased isoflavonoid consumption are a matter of continuing investigation and debate [Miadoková, 2009], it has received specific study for its role in cancer prevention. There are multiple proposed mechanisms other than antioxidant activity by which isoflavonoids may contribute directly to cancer prevention. These include induction of cell cycle arrest and apoptosis, induction of detoxification enzymes, regulation of host immune system, and changes in cellular signaling [Ito et al., 2006; Birt et al., 2001]. It is therefore feasible that our observed increases in phenolics within chickpeas during sprouting may be contributing health benefits other than 
TABLE 2. Descriptive analysis of pasta samples with different levels of chickpea flour substitutiona.

\begin{tabular}{|c|c|c|c|c|c|c|}
\hline & Attribute & $100 \%$ Semolina & $20 \% \mathrm{NSCF}^{\mathrm{b}}$ & $40 \% \mathrm{NSCF}^{\mathrm{b}}$ & $20 \% \mathrm{SCF}^{b}$ & $40 \% \mathrm{SCF}^{\mathrm{b}}$ \\
\hline \multirow{3}{*}{ 节 } & Chewiness & $0.62^{\mathrm{A}}$ & $0.53^{\mathrm{AB}}$ & $0.26^{\mathrm{C}}$ & $0.45^{\mathrm{AB}}$ & $0.35^{\mathrm{BC}}$ \\
\hline & Mushiness & $0.24^{\mathrm{B}}$ & $0.28^{\mathrm{AB}}$ & $0.48^{\mathrm{A}}$ & $0.29^{\mathrm{AB}}$ & $0.43^{\mathrm{A}}$ \\
\hline & Grittiness & $0.08^{\mathrm{B}}$ & $0.21^{\mathrm{A}}$ & $0.19^{\mathrm{A}}$ & $0.21^{\mathrm{A}}$ & $0.26^{\mathrm{A}}$ \\
\hline \multirow{3}{*}{ 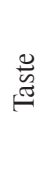 } & Saltiness & $0.06^{\mathrm{B}}$ & $0.09^{\mathrm{AB}}$ & $0.14^{\mathrm{A}}$ & $0.11^{\mathrm{AB}}$ & $0.13^{\mathrm{A}}$ \\
\hline & Sweetness & $0.25^{\mathrm{A}}$ & $0.31^{\mathrm{A}}$ & $0.20^{A}$ & $0.23^{\mathrm{A}}$ & $0.19^{\mathrm{A}}$ \\
\hline & Bitterness & $0.06^{\mathrm{C}}$ & $0.12^{\mathrm{BC}}$ & $0.17^{\mathrm{AB}}$ & $0.28^{\mathrm{A}}$ & $0.28^{\mathrm{A}}$ \\
\hline \multirow{2}{*}{ 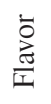 } & Earthiness & $0.10^{\mathrm{C}}$ & $0.33^{\mathrm{B}}$ & $0.30^{\mathrm{B}}$ & $0.37^{\mathrm{B}}$ & $0.57^{\mathrm{A}}$ \\
\hline & Pasta flavor & $0.61^{\mathrm{A}}$ & $0.45^{\mathrm{AB}}$ & $0.38^{\mathrm{B}}$ & $0.32^{\mathrm{BC}}$ & $0.22^{\mathrm{C}}$ \\
\hline
\end{tabular}

aDetermined by descriptive analysis by eight trained panelists. All panelists assessed each sample at three distinct evaluation sessions. Results reported

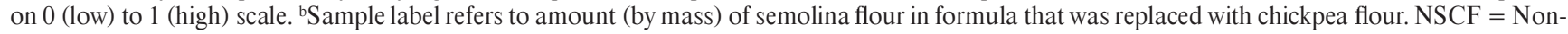
-Sprouted Chickpea Flour; SCF = Sprouted Chickpea Flour. Values followed by the same superscript capital letter within a row were not significantly different from one another $(\alpha=0.05)$ according to ANOVA and means separation with Tukey's Studentized Range via SAS software.

TABLE 3. Predictive modeling of descriptive analysis results according to chickpea flour substitution levels ${ }^{\mathrm{a}}$.

\begin{tabular}{|c|c|c|c|c|c|}
\hline & \multirow{2}{*}{ Attribute } & \multirow{2}{*}{ Intercept } & \multicolumn{2}{|c|}{ Coefficients } & \multirow{2}{*}{$\mathrm{R}^{2}$ (Adj.) } \\
\hline & & & $\operatorname{NSCF}(\%)^{\mathrm{b}}$ & $\operatorname{SCF}(\%)^{\mathrm{b}}$ & \\
\hline \multirow{3}{*}{ 氖 } & Chewiness & 0.636 & -0.00857 & -0.00757 & $82.5 \%$ \\
\hline & Mushiness & 0.204 & 0.00627 & 0.00537 & $77.3 \%$ \\
\hline & Grittiness & 0.0933 & 0.00275 & 0.00450 & $61.6 \%$ \\
\hline \multirow{3}{*}{ 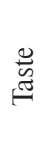 } & Saltiness & 0.0614 & 0.00186 & 0.00186 & $87.5 \%$ \\
\hline & Sweetness & \multicolumn{4}{|c|}{ no significant variables ${ }^{\mathrm{c}}$} \\
\hline & Bitterness & 0.127 & 0.00275 & 0.00459 & $81.2 \%$ \\
\hline \multirow{2}{*}{ 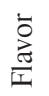 } & Earthiness & 0.147 & 0.00489 & 0.0107 & $79.8 \%$ \\
\hline & Pasta flavor & 0.570 & -0.00500 & -0.00950 & $84.6 \%$ \\
\hline
\end{tabular}

${ }^{a}$ Models based upon sensory scores determined by descriptive analysis by eight trained panelists. All panelists assessed each sample at three distinct evaluation sessions. Results were reported on 0 (low) to 1 (high) scale. ${ }^{b}$ Percentage of chickpea flour used in substitution of semolina flour in pasta formula. NSCF $=$ Non-Sprouted Chickpea Flour; SCF $=$ Sprouted Chickpea Flour. 'Variables with $\mathrm{p}<0.10$ were excluded from reported models. Linear regression models made with SAS software.

radical quenching. To this point, a recent in vivo investigation determined that consumption of sprouted chickpea pasta resulted in greater brachial artery flow mediated dilation than consumption of semolina flour pasta [Enrique et al., 2018].

Our results suggest that sprouting chickpeas increases the concentration of antioxidant compounds and in vitro antioxidant potential, and provide evidence of a possible consistency in the approximate magnitude of these changes. The results also affirm that this improvement of antioxidant contents and potential is present within the samples exposed in this study to sensory evaluation. Further research into the composition differences (and the associated in vivo effects) between SCF and NSCF may be warranted to further elucidate the mechanism of the observed changes.

\section{Descriptive analysis}

The results of the descriptive analysis are shown in Table 2 and the predictive models for these attributes according to substitution are shown in Table 3. Figure 1 depicts descriptive characteristics of the $100 \%$ semolina sample alongside the average value for the two NSCF samples (20 and 40\% substitution) and the average value for the two SCF samples (20 and 40\% substitution). Regarding textural attributes, the data suggests that substitution of semolina with chickpea flour at levels of $40 \%$ results in reductions in chewiness and increases in mushiness and grittiness. For all three of the assessed textural attributes, both $40 \%$ chickpea flour substitutions were significantly different from the $100 \%$ semolina sample. Notably, though, in none of the textural assessments were significant differences found between the $40 \%$ NSCF and $40 \%$ SCF samples. Examination of the coefficients of the predictive models indicate decreases in chewiness and increases in mushiness and grittiness with greater levels of chickpea flour substitution. The coefficients of the models suggest greater magnitude of the effect associated with NSCF for chewiness and mushiness, and with SCF for grittiness. 


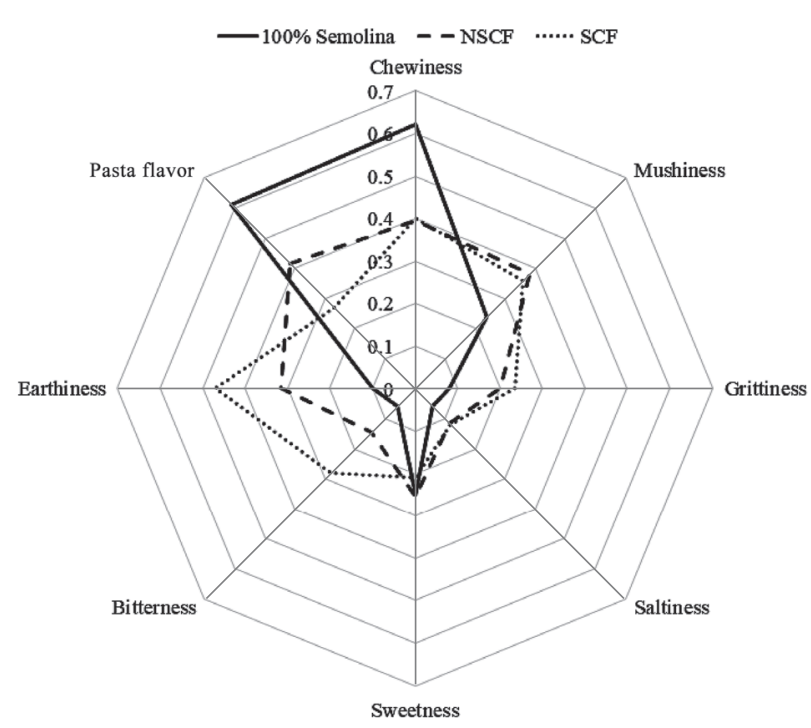

FIGURE 1. Descriptive analysis of pasta samples with non-sprouted and sprouted chickpea flour substitution ${ }^{\mathrm{ab}}$.

aDetermined by descriptive analysis by eight trained panelists. All panelists assessed each sample at three distinct evaluation sessions. Results reported on 0 (low) to 1 (high) scale. ${ }^{b}$ " $100 \%$ Semolina" had no substitution; "NSCF" is average value for samples of $20 \%$ and $40 \%$ substitution non-sprouted chickpea flour; "SCF" is average value for samples of $20 \%$ and $40 \%$ substitution sprouted chickpea flour.

The loss of chewiness and increases in mushiness and grittiness would all likely suggest quality impairment, and these effects correspond well to documented challenges with the substitution of gluten-free flours [Kohajdová et al., 2011; Wood, 2009]. Our data suggests, however, that these challenges may not be exacerbated by the use of SCF rather than NSCF.

Regarding tastes, the data shows no significant differences between any samples for sweetness, and neither chickpea flour was a significant variable for this output in the models. For bitterness, 40\% NSCF, 20\% SCF, and 40\% SCF each had significantly higher values than the $100 \%$ semolina sample $(p=0.0054, p=0.0001$, and $p=0.0001$, respectively). None of these three samples, however, were significantly different from one another. NSCF and SCF were both significant positive predictors of bitterness in the models, with the coefficient of SCF 1.6 times greater than that of NSCF. Increases in bitterness associated with chickpea flour incorporation has been shown in a study previously [Kohajdová et al., 2011], and our study indicates this effect may be more noticeable when using $\mathrm{SCF}$ rather than NSCF.

Interestingly, our data show significant increases in perceptions of saltiness at a substitution level of $40 \%$ for both NSCF and SCF, and both variables were determined to be positively associative in the predictive models. To the knowledge of the authors, this particular effect has not been previously documented, but this perception of the panelists may feasibly be due to increases in other tastes and flavors associated with the chickpea flour. This suggests that the use of chickpea flour in place of semolina may allow for salt reduction in formulation.

Regarding flavors, all samples with chickpea flour had significantly greater earthiness flavor than $100 \%$ semolina. $40 \%$ NSCF, 20\% SCF, and 40\% SCF each had significantly lower pasta flavor than $100 \%$ semolina. For both assessed flavor attributes, $40 \%$ SCF was significantly different from either NSCF sample, and the models for both attributes show a greater magnitude of coefficient for SCF than for NSCF. The data suggests that these flavor changes are more substantial when substituting SCF rather than NSCF for semolina flour in pasta.

A prior study [Rayas-Duarte et al., 1996] that investigated buckwheat substitution in pasta (and examined two of the same sensory attributes as our own study) helps us to contextualize the magnitude of our observed effects. Specifically, our study found that $40 \%$ SCF resulted in a $325 \%$ increase in grittiness and $570 \%$ increase in earthiness vs. the control. The study on buckwheat flour determined that $30 \%$ dark buckwheat substitution resulted in 1,467\% increase in grittiness and a $300 \%$ increase in earthiness vs. the control. So although our data indicates significant sensory effects are associated with the substitution of SCF, effects of similar and greater magnitude have been observed for other health-promoting flour substitutions in pasta.

\section{Consumer assessment}

The results of the consumer assessments are shown in Table 4. For the measure of appearance, $40 \%$ NSCF was the only sample that differed significantly from the $100 \%$ semolina sample $(p=0.0246)$. The deterioration of appearance in pasta with NSCF substitution levels exceeding $20 \%$ has been shown once previously in lasagna noodles [Sabanis et al., 2006]. Our results indicate that this organoleptic challenge may be diminished when substituting SCF rather than NSCF.

TABLE 4. Consumer assessments of pasta samples with different levels of chickpea flour substitution ${ }^{\mathrm{a}}$

\begin{tabular}{lccc|c|c}
\hline Hedonic measure & $100 \%$ Semolina & $20 \% \mathrm{NSCF}^{\mathrm{b}}$ & $40 \% \mathrm{NSCF}^{\mathrm{b}}$ & $20 \% \mathrm{SCF}^{\mathrm{b}}$ & $40 \% \mathrm{SCF}^{\mathrm{b}}$ \\
\hline Appearance & $4.38^{\mathrm{A}}$ & $4.27^{\mathrm{A}}$ & $3.93^{\mathrm{B}}$ & $4.36^{\mathrm{A}}$ & $4.63^{\mathrm{A}}$ \\
Texture & $4.67^{\mathrm{A}}$ & $4.70^{\mathrm{A}}$ & $4.36^{\mathrm{A}}$ & $4.55^{\mathrm{A}}$ & $4.41^{\mathrm{A}}$ \\
Flavor & $5.05^{\mathrm{A}}$ & $4.57^{\mathrm{B}}$ & $4.36^{\mathrm{B}}$ & $4.56^{\mathrm{B}}$ & $3.90^{\mathrm{C}}$ \\
Overall & $4.86^{\mathrm{A}}$ & $4.48^{\mathrm{BC}}$ & $4.51^{\mathrm{ABC}}$ & $4.63^{\mathrm{AB}}$ & $4.25^{\mathrm{C}}$ \\
\hline
\end{tabular}

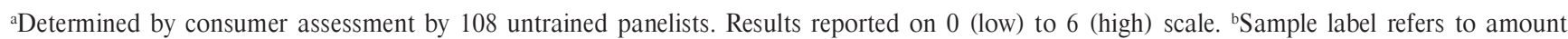
(by mass) of semolina flour in formula that was replaced with chickpea flour. NSCF $=$ Non-Sprouted Chickpea Flour; SCF $=$ Sprouted Chickpea Flour. Values followed by the same superscript capital letter within a row were not significantly different from one another $(\alpha=0.05)$ according to ANOVA and means separation with Tukey's Studentized Range via SAS software. 
Despite the descriptive results indicating loss of chewiness and increases in mushiness and grittiness with chickpea flour substitution, the hedonic assessments showed no significant differences in texture between any of the samples. Wood [2009] previously found that substitution with NSCF reduced pasta firmness, but still resulted in acceptable product quality. Our results indicate this effect to not be different when using SCF rather than NSCF.

The most substantial observed changes in hedonic measures occurred with flavor, for which all chickpea flour samples performed significantly worse than the $100 \%$ semolina sample. Moreover, the $40 \%$ SCF samples were significantly worse than all other samples in this regard. This observation corresponds well with the descriptive analysis results which indicated SCF to more substantially influence earthiness (positive association) and pasta flavor (negative association) than NSCF. The positive associative effect of SCF on bitterness (although technically a taste) may also have contributed to the flavor assessment. The results suggest that SCF may present greater challenges to flavor quality than NSCF. It is worth noting, though, that at $20 \%$ substitution, the SCF sample was not significantly different from NSCF. Therefore, this separation in effect may only occur at relatively high substitution levels (exceeding 20\%).

In the overall hedonic assessment, $40 \%$ SCF was the only sample to differ significantly from $100 \%$ semolina $(p=0.0007)$. As with flavor, the results suggest that SCF substitution may present more organoleptic challenges than NSCF substitution, but that this distinction may only be present with substitution levels exceeding $20 \%$.

\section{CONCLUSIONS}

Sprouting of chickpeas prior to flour production can increase phenolic contents and in vitro antioxidant potential. However, sensory evaluation indicates that the use of SCF rather than NSCF may present some challenges to product quality - particularly regarding bitterness and effects on flavor. Notably, this distinction between chickpea flour types may only occur at substitution levels in excess of $20 \%$. Considering the observed increases in healthful components, SCF may merit consideration as an alternative to NSCF in bulk production and product formulation, but attention must be given to the effects on flavor and taste quality that may occur at high levels of substitution. Further studies investigating methods to mitigate these quality concerns may be warranted.

\section{CONFLICT OF INTEREST}

The authors declare that they have no conflict of interest.

\section{REFERENCES}

1. ASTM International. (2011). Standard Guide for Sensory Evaluation Methods to Determine the Sensory Shelf Life of Consumer Products. ASTM International.

2. Birt, D.F., Hendrich, S., Wang, W. (2001). Dietary agents in cancer prevention: flavonoids and isoflavonoids. Pharmacology \& Therapeutics, 90(2-3), 157-177.
3. Bouchenak, M., Lamri-Senhadji, M. (2013). Nutritional quality of legumes, and their role in cardiometabolic risk prevention: a review. Journal of Medicinal Food, 16(3), 185-198.

4. Brand-Williams, W., Cuvelier, M.-E., Berset, C. (1995). Use of a free radical method to evaluate antioxidant activity. LWT-Food Science and Technology, 28(1), 25-30.

5. Devi, C.B., Kushwaha, A., Kumar, A. (2015). Sprouting characteristics and associated changes in nutritional composition of cowpea (Vigna unguiculata). Journal of Food Science and Technology - Mysore, 52(10), 6821-6827.

6. Enrique, A., Bruno, J., Feldman, C., Kerrihard, A., Matthews, E. (2018). Effects of germinated chickpea flour incorporated in pasta on brachial artery flow mediated dilation. International Journal of Exercise Science - Conference Proceedings, 9(6), 1.

7. FMI (2018). Chickpea Flour Market: Global Industry Analysis, Size and Forecast, 2017 to 2026. Retrieved from [https://www. futuremarketinsights.com/reports/chickpea-flour-market].

8. Gao, Y., Yao, Y., Zhu, Y., Ren, G. (2015). Isoflavone content and composition in chickpea (Cicer arietinum L.) sprouts germinated under different conditions. Journal of Agricultural and Food Chemistry, 63(10), 2701-2707.

9. Gharachorloo, M., Ghiassi Tarzi, B., Baharinia, M. (2013). The effect of germination on phenolic compounds and antioxidant activity of pulses. Journal of the American Oil Chemists' Society, 90(90), 407-411.

10. Gunashree, B.S., Kumar, R.S., Roobini, R., Venkateswaran, G. (2014). Nutrients and antinutrients of ragi and wheat as influenced by traditional processes. International Journal of Current Microbiology and Applied Sciences, 3(7), 720-736.

11. Gupta, R.K., Gupta, K., Sharma, A., Das, M., Ansari, I.A., Dwivedi, P.D. (2017). Health risks and benefits of chickpea ( $\mathrm{Ci}$ cer arietinum) consumption. Journal of Agricultural and Food Chemistry, 65(1), 6-22.

12. Ito, C., Murata, T., Itoigawa, M., Nakao, K., Kumagai, M., Kaneda, N., Furukawa, H. (2006). Induction of apoptosis by isoflavonoids from the leaves of Millettia taiwaniana in human leukemia HL-60 cells. Planta Medica, 72, 424-429.

13. Khattak, A.B., Zeb, A., Khan, M., Bibi, N., Khattak, M.S. (2007). Influence of germination techniques on sprout yield, biosynthesis of ascorbic acid and cooking ability, in chickpea (Cicer arietinum L.). Food Chemistry, 103(1), 115-120.

14. Kohajdová, Z., Karovicova, J., Magala, M. (2011). Utilisation of chickpea flour for crackers production. Acta Chimica Slovaca, 4, 98-107.

15. Kumar, M., Hora, R., Kostrzynska, M., Waites, W.M., Warriner, K. (2006). Inactivation of Escherichia coli O157: H7 and Salmonella on mung beans, alfalfa, and other seed types destined for sprout production by using an oxychloro-based sanitizer. Journal of Food Protection, 69(7), 1571-1578.

16. Masood, T., Shah, H.U., Zeb, A. (2014). Effect of sprouting time on proximate composition and ascorbic acid level of mung bean (Vigna radiate L.) and chickpea (Cicer arietinum L.) seeds. Journal of Animal and Plant Sciences, 24(3), 850-859.

17. Meilgaard, M.C., Carr, B.T., Civille, G.V. (1999). Sensory Evaluation Techniques. CRC Press Boca Raton, Florida, USA.

18. Melini, F., Melini, V., Luziatelli, F., Ruzzi, M. (2017). Current and forward-looking approaches to technological and nutritional improvements of gluten-free bread with legume flours: 
A critical review. Comprehensive Reviews in Food Science and Food Safety, 16(5), 1101-1122.

19. Miadoková, E. (2009). Isoflavonoids-an overview of their biological activities and potential health benefits. Interdisciplinary Toxicology, 2(4), 211-218.

20. Nakitto, A.M., Muyonga, J.H., Nakimbugwe, D. (2015). Effects of combined traditional processing methods on the nutritional quality of beans. Food Science \& Nutrition, 3(3), 233-241.

21. Padalino, L., Mastromatteo, M., Lecce, L., Spinelli, S., Conte, A., Alessandro Del Nobile, M. (2015). Optimization and characterization of gluten-free spaghetti enriched with chickpea flour. International Journal of Food Sciences and Nutrition, 66(2), 148-158.

22. Polak, R., Phillips, E.M., Campbell, A. (2015). Legumes: Health benefits and culinary approaches to increase intake. Clinical Diabetes, 33(4), 198-205.

23. Ramesh, D.R., Swami, S.C. (2016). Total antioxidant capacity of some common seeds and effect of sprouting and its health benefits. International Journal of Chemical Studies, 4(2), 25-27.

24. Rayas-Duarte, P., Mock, C.M., Satterlee, L.D. (1996). Quality of spaghetti containing buckwheat, amaranth, and lupin flours. Cereal Chemistry, 73(3), 381-387.

25. Sabanis, D., Makri, E., Doxastakis, G. (2006). Effect of durum flour enrichment with chickpea flour on the characteristics of dough and lasagne. Journal of the Science of Food and Agriculture, 86(12), 1938-1944.

26. Segev, A., Badani, H., Galili, L., Hovav, R., Kapulnik, Y., Shomer, I., Galili, S. (2011). Total phenolic content and antioxidant activity of chickpea (Cicer arietinum L.) as affected by soaking and cooking conditions. Food and Nutrition Sciences, 2, 724.

27. Singleton, V.L., Orthofer, R., Lamuela-Raventós, R.M. (1999). Analysis of total phenols and other oxidation substrates and antioxidants by means of Folin-Ciocalteu reagent. In J. Abelson (Ed.), Methods in Enzymology, Vol. 299, Elsevier, Amsterdam, The Netherlands, pp. 152-178.
28. Stone, N.J., Robinson, J.G., Lichtenstein, A.H., Bairey Merz, C.N., Blum, C.B., Eckel, R.H., Goldberg A.C., Gordon D., Levy D., Lloyd-Jones, D.M. (2014). American Heart Association Task Force on Practice G. 2013 ACC/AHA guideline on the treatment of blood cholesterol to reduce atherosclerotic cardiovascular risk in adults: A report of the American College of Cardiology/ American Heart Association Task Force on Practice Guidelines. Circulation, 129(25), S1-S45.

29. Świeca, M., Gawlik-Dziki, U., Jakubczyk, A. (2013). Impact of density of breeding on the growth and some nutraceutical properties of ready-to-eat lentil (Lens culinaris) sprouts. Acta Scientiarum Polonorum Hortorum Cultus, 12(4), 19-29.

30. Venn, B.J., Perry, T., Green, T.J., Skeaff, C.M., Aitken, W., Moore, N.J., Mann J.I., Wallace A.J., Monro J., Bradshaw A., Brown R.C., Skidmore P.M.L., Doel K., O’Brien K., Frampton C., Williams, S. (2010). The effect of increasing consumption of pulses and wholegrains in obese people: A randomized controlled trial. Journal of the American College of Nutrition, 29(4), 365-372.

31. Wood, J.A. (2009). Texture, processing and organoleptic properties of chickpea-fortified spaghetti with insights to the underlying mechanisms of traditional durum pasta quality. Journal of Cereal Science, 49(1), 128-133.

32. Wu, Z.Y., Song, L.X., Feng, S.B., Liu, Y.C., He, G.Y., Yioe, Y., Liu S.Q., Huang, D. (2012). Germination dramatically increases isoflavonoid content and diversity in chickpea (Cicer arietinum L.) seeds. Journal of Agricultural and Food Chemistry, 60(35), 8606-8615.

33. Zafar, T.A., Al-Hassawi, F., Al-Khulaifi, F., Al-Rayyes, G., Waslien, C., Huffman, F.G. (2015). Organoleptic and glycemic properties of chickpea-wheat composite breads. Journal of Food Science and Technology - Mysore, 52(4), 2256-2263.

Submitted: 5 March 2019. Revised: 26 April 2019. Accepted: 10 May 2019. Published on-line: 31 May 2019. 